TTR

Traduction, terminologie, rédaction

\title{
De l'emploi des surtitres anglais dans les théâtres franco-canadiens : bénéfice et préjudice
}

\section{Louise Ladouceur}

Volume 28, numéro 1-2, 1er semestre-2e semestre 2015

La traduction littéraire et le Canada

Literary translation and Canada

URI : https://id.erudit.org/iderudit/1041658ar

DOI : https://doi.org/10.7202/1041658ar

Aller au sommaire du numéro

Éditeur(s)

Association canadienne de traductologie

ISSN

0835-8443 (imprimé)

1708-2188 (numérique)

Découvrir la revue

Citer cet article

Ladouceur, L. (2015). De l'emploi des surtitres anglais dans les théâtres franco-canadiens : bénéfice et préjudice. TTR, 28(1-2), 239-257.

https://doi.org/10.7202/1041658ar

\section{Résumé de l'article}

Cet article propose une étude du surtitrage dans les théâtres francophones de l'Ouest canadien afin de mettre en relief ce qui distingue ce mode de traduction dans le champ de la traduction audiovisuelle. Le théâtre étant un art vivant, les surtitres doivent composer avec la nature instable du texte source livré sur scène et les différents profils linguistiques des destinataires auxquels ils sont transmis pendant le spectacle. Mis en pratique depuis plusieurs années dans les théâtres franco-canadiens, le surtitrage offre plusieurs avantages aux compagnies théâtrales fonctionnant dans une langue française minorisée puisqu'il permet à l'oeuvre de rejoindre un public élargi tout en conservant sa spécificité linguistique et esthétique. Toutefois, parce qu’il oblige les langues source et cible à cohabiter dans un espace traditionnellement consacré à des productions culturelles d'expression française, ce mode de traduction remet en cause la vocation des théâtres francophones en contexte minoritaire. Enfin, devenue pratique courante dans les théâtres franco-canadiens, le surtitrage demeure absent des scènes anglophones, ce qui met en relief l'asymétrie des langues officielles du Canada.
Ce document est protégé par la loi sur le droit d'auteur. L'utilisation des services d’Érudit (y compris la reproduction) est assujettie à sa politique d'utilisation que vous pouvez consulter en ligne.

https://apropos.erudit.org/fr/usagers/politique-dutilisation/ 


\title{
De l'emploi des surtitres anglais dans les théâtres franco-canadiens : bénéfice et préjudice
}

\author{
Louise Ladouceur \\ Université de l'Alberta
}

\section{Résumé}

Cet article propose une étude du surtitrage dans les théâtres francophones de l'Ouest canadien afin de mettre en relief ce qui distingue ce mode de traduction dans le champ de la traduction audiovisuelle. Le théâtre étant un art vivant, les surtitres doivent composer avec la nature instable du texte source livré sur scène et les différents profils linguistiques des destinataires auxquels ils sont transmis pendant le spectacle. Mis en pratique depuis plusieurs années dans les théâtres franco-canadiens, le surtitrage offre plusieurs avantages aux compagnies théâtrales fonctionnant dans une langue française minorisée puisqu'il permet à l'œuvre de rejoindre un public élargi tout en conservant sa spécificité linguistique et esthétique. Toutefois, parce qu'il oblige les langues source et cible à cohabiter dans un espace traditionnellement consacré à des productions culturelles d'expression française, ce mode de traduction remet en cause la vocation des théâtres francophones en contexte minoritaire. Enfin, devenue pratique courante dans les théâtres franco-canadiens, le surtitrage demeure absent des scènes anglophones, ce qui met en relief l'asymétrie des langues officielles du Canada.

Mots-clés: surtitres, traduction audiovisuelle, théâtre franco-canadien

\section{Abstract}

This article focuses on surtitling in francophone theatres of Western Canada in order to illustrate how this translation modality distinguishes itself in the field of audio-visual translation. Theatre being a living art, surtitles must adapt to the instable nature of the source text delivered on stage and take into account the various linguistic profiles of the audience. Adopted by Franco-Canadian theatres, surtitling offers many advantages to companies working in a minority language as it renders the production accessible to a larger audience while retaining its linguistic and aesthetic specificity. However, since it imposes a cohabitation of the source and target languages in a space traditionally dedicated to promote and defend francophone cultural products, this translation mode can be prejudicial to the vocation of francophone theatre companies in a minority context. Now firmly established 
in Franco-Canadian theatres, surtitles remain absent in anglophone theatres thus underlining the asymmetry between the official languages of Canada.

Keywords: surtitles, audiovisual translation, Franco-Canadian theatre

Cet article propose une étude des fonctions et de la réception des surtitres dans les théâtres francophones de l'Ouest canadien afin de mettre en relief ce qui distingue ce mode de traduction dans le champ de la traduction audiovisuelle. À la différence des produits audiovisuels transmis au cinéma, à la télévision ou sur Internet, la production théâtrale est un spectacle vivant qui met en scène des artistes et un public physiquement présents pendant la représentation. Parmi les arts vivants, le théâtre est celui qui entretient les liens les plus étroits avec la communauté à laquelle il s'adresse, ce qui génère des attentes particulières de la part du public. Les spécificités de la communication théâtrale agissent sur les modalités de transmission et de réception de la traduction d'une façon qui appartient en propre au théâtre.

Comparables aux sous-titres utilisés au cinéma, les surtitres offrent une traduction simultanée des dialogues livrés sur scène pendant une représentation théâtrale. Ils peuvent être projetés sur des écrans près de la scène, intégrés aux sièges dans la salle, transmis sur des tablettes numériques ou intégrés à des lunettes à réalité augmentée (voir Laliberté, 2017). Inauguré au Canada en 1983 par la Canadian Opera Company, le surtitrage a rapidement été mis à profit par plusieurs festivals de théâtre internationaux, qui ont pu ainsi présenter des productions en plusieurs langues accompagnées de surtitres adaptés aux publics visés. Il s'est par la suite imposé dans divers théâtres franco-canadiens, qui produisent leurs spectacles en saison régulière avec des surtitres anglais. Le modèle, mis en place en $2005 \mathrm{au}$ Théâtre français de Toronto, a été adopté par la Troupe du Jour de Saskatoon en 2007, par L'UniThéâtre d'Edmonton et le Théâtre de la Seizième à Vancouver en 2008 et par le Cercle Molière de Winnipeg en 2014.

Le surtitrage anglais offre plusieurs avantages aux petits théâtres fonctionnant dans une langue minorisée puisqu'il permet à leurs spectacles de rejoindre un public élargi tout en conservant leur spécificité linguistique et esthétique. Son usage comporte toutefois un certain risque en ce qu'il peut contribuer à la fragilisation du français, qui se voit obligé de cohabiter avec l'anglais dans un espace traditionnellement réservé à la culture 
d'expression française. Absent des scènes anglo-canadiennes, le surtitrage s'est imposé sur les scènes des compagnies œuvrant dans une langue minoritaire, désireuses de rejoindre le public plus large des locuteurs de la langue dominante. Ce mode de traduction à sens unique illustre l'asymétrie du rapport qu'entretiennent les langues officielles du Canada ${ }^{1}$.

\section{Traduction audiovisuelle et surtitrage théâtral}

Selon Yves Gambier, la traduction audiovisuelle s'est constituée en champ d'études dans le domaine de la traductologie au milieu des années 1990 (2004, p. 1). Elle incluait alors le sous-titrage et le doublage d'œuvres destinées à l'écran (cinéma, télévision, etc.), la traduction de services et produits en ligne ainsi que le surtitrage pour l'opéra. Avec l'apparition des surtitres dans plusieurs théâtres européens au courant des années 1990, un débat s'est engagé sur la pertinence d'intégrer le surtitrage pour le théâtre dans le champ de la traduction audiovisuelle. Il a fallu alors établir une distinction entre texte dramatique et texte théâtral: «[b]y dramatic text we understand the written text, as having been conceived for the theatre, whilst we use the term theatrical text when the staging in the theatre is being referred to" (Bartoll, 2011, p. 88). Cette distinction est en effet cruciale, car chaque type de texte fait appel à des modalités de traduction qui ont peu en commun. J'emprunterai ces catégories pour les appliquer à la traduction.

Alors que la traduction du texte dramatique (traduction dramatique) est un produit autonome qui existe indépendamment du texte source, la traduction audiovisuelle du texte théâtral (traduction théâtrale) n'existe qu'en fonction de la performance: « $[\mathrm{t}]$ he translation cannot be read as an independent text because it forms an integral part of the staging" (Dewolf citée par Bartoll, 2011, p. 89). Ici, la notion de performability, vivement débattue dans les années 1980, acquiert une nouvelle valeur. Susan Bassnett affirme que le terme est «frequently used to describe the indescribable $[\ldots]$. There is no sound theoretical base for arguing that "performability" can or does exist» (1991, p. 102). Elle précise toutefois :

Theatre texts cannot be considered as identical to texts written to be read because the process of writing involves

1. Pour une étude du rapport de force à l’œuvre dans les traductions théâtrales au Canada, voir Ladouceur (2005). 
a consideration of the performance dimension, but neither can an abstract notion of performance be put before textual considerations» (ibid., p. 110).

L'emploi des surtitres au théâtre permet de revisiter la notion de performability en l'appliquant non plus au texte écrit, traduit pour être joué, mais au texte joué, traduit pour être lu pendant la performance. Il ne s'agirait plus d'une notion abstraite, inhérente au texte et antérieure à la traduction, mais plutôt du résultat concret de l'opération traduisante soumise à l'épreuve de la performance, qui pourrait se mesurer à la manière dont elle façonne le texte actualisé par l'interprète selon une mise en scène ${ }^{2}$ donnée, destinée à un public donné. La traduction théâtrale donnerait ainsi à voir la "performabilité» du texte en traduction, conditionnée et déterminée par la "performance» à laquelle il participe.

En effet, bien qu'il ait préalablement existé sous forme écrite, le texte faisant l'objet de la traduction est le texte oral livré sur scène pendant le spectacle. Il s'agit d'un texte façonné par divers facteurs paralinguistiques tels que la mise en scène, l'interprétation des acteurs ainsi que les signes visuels et auditifs contribuant au spectacle. La traduction du texte joué peut aussi être appelée à varier selon les destinataires visés et les fonctions attribuées à la traduction. Ainsi, les sacres et les jurons apparaissant dans les surtitres d'un spectacle grand public pourront faire l'objet de révisions lorsque le même spectacle est présenté à un auditoire plus jeune dans le cadre d'un exercice pédagogique. Les procédés de traduction pourront aussi varier selon les profils linguistiques - unilingues ou multilingues - et les langues privilégiées de l'auditoire visé. Ainsi, pour un spectacle présenté en tournée, il suffira de modifier au besoin certains surtitres, d'en enlever ou d'en insérer de nouveaux pour ajuster les dialogues selon les variations dialectales propres aux publics ciblés.

Le théâtre étant un art vivant ${ }^{3}$ requérant la présence physique d'artistes qui se produisent devant un public, le texte livré sur scène pendant le spectacle peut changer au gré des représentations de

2. Patrice Pavis insiste sur l'importance de la mise en scène dans la traduction théâtrale: «translation for the stage borrows means other than those of a purely linguistic translation and [...] takes place on the level of the mise en scène as a whole» $(1989$, p. 41).

3. Les arts vivants incluent le théâtre, la danse, les arts du cirque, les arts de la rue, les arts de la marionnette, l'opéra et la musique live. 
façons imprévisibles. Cette instabilité du texte source est propre à la traduction appliquée aux arts vivants, et plus particulièrement au théâtre. À la différence de l'opéra, dont les dialogues suivent le déroulement de la partition musicale, le texte de théâtre peut fluctuer suite à des improvisations, des changements de rythme, des trous de mémoire ou autres variations dans la performance. Qui plus est, alors que le livret d'opéra est rédigé dans un style lyrique et est destiné à être chanté, les dialogues de théâtre sont conçus pour être livrés dans une langue parlée qui semble naturelle et sonne juste. Selon Jonathan Burton, les surtitres utilisés à l'opéra «are trying to convey what is being said, not how it is being said» (2010, p. 184). Cela ne saurait s'appliquer au théâtre, car les spectateurs doivent suivre les dialogues de près afin de recueillir l'information nécessaire pour comprendre l'action. Malgré l'instabilité du texte oral livré sur scène, le surtitre théâtral doit viser une grande fidélité à son contenu et à sa forme. Il doit tenir compte, par exemple, des différences dans les niveaux de langue, d'un idiolecte particulier, des accents et de toute particularité de la langue orale qui véhicule une information pertinente dans le cadre du spectacle. À la différence du texte d'opéra, le texte théâtral est marqué par une instabilité qui fait en sorte que sa traduction n'est jamais arrêtée, c'est une traduction "vivante», en constant devenir. Pendant le spectacle ou entre les représentations, la traduction est corrigée selon les besoins de la performance ou de l'auditoire visé. Cette distinction entre les surtitres pour l'opéra et pour le théâtre mérite une étude plus détaillée qui reste à faire.

\section{Traduction théâtrale, avantages et contraintes}

Pour les théâtres œuvrant en contexte minoritaire, les surtitres offrent de nombreux avantages. En premier lieu, ils leur permettent de rejoindre un auditoire élargi incluant des locuteurs d'autres langues. Les théâtres franco-canadiens peuvent ainsi avoir accès au vaste public anglophone unilingue qui compose la majorité de la population à l'ouest du Québec, ce qui augmente leur rentabilité et leur visibilité. Dans le cas du Théâtre français de Toronto, "plusieurs études ont démontré que le français neétait pas la langue maternelle de près d'un quart de son public et cette proportion peut atteindre $55 \%$ lors des représentations avec surtitres en anglais» (site web de l'Association des théâtres francophones du Canada [ATFC]). Pour la Troupe du Jour de Saskatoon, dès l'apparition des surtitres en 2007, «le taux d'occupation de la salle 
lors des représentations avec les surtitres était en moyenne $16 \%$ plus élevé que lors des représentations sans les surtitres» (site web de l'ATFC). Un sondage effectué au même théâtre en octobre 2006 pour la production de Bonbons assortis de Michel Tremblay "a révélé qu'environ 300 personnes étaient venues voir la pièce grâce aux surtitres et que trois quarts d'entre elles reconnaissaient avoir besoin de ce procédé pour suivre un spectacle en français» (site web de l'ATFC).

Autre avantage de taille, le surtitrage rend une production accessible à divers auditoires tout en préservant la spécificité linguistique et culturelle du spectacle. Plutôt que d'effacer les codes linguistiques et culturels du texte source comme c'est le cas avec la traduction conventionnelle, qui évacue le texte source au profit du texte cible, le surtitrage les donne à voir et à entendre aux spectateurs pour qui les dialogues seraient autrement inintelligibles. Dans des sociétés de plus en plus multilingues et multiculturelles, cela constitue un formidable vecteur d'échanges culturels, comme le souligne Linda Dewolf:

[1]e pari linguistique et culturel qu'il [le surtitrage] pose vise à préserver l'identité d'une communauté et la place préservée de chaque patrimoine culturel en procurant, au-delà du divertissement et du spectacle visuel, une découverte esthétique, une jouissance intellectuelle et une palette d'émotions qui nous incitent à découvrir un nouveau langage théâtral, un jeu et une écriture. (2003, p. 106)

Aménageant des pistes pour scruter les rapports entre les cultures et les langues, le surtitrage théâtral est un «terrain de prédilection pour éprouver des hypothèses et tenter des expériences d'interculturalisme»(ibid.). En ce sens, le recours au surtitrage peut dépasser sa visée première et devenir un outil de créativité artistique dans l'exploration des espaces hétérolingues et interculturels qui composent nos sociétés multiethniques et qui sont le propre des communautés linguistiques minoritaires.

Enfin, les surtitres sont un mode de traduction souple et économique. Il existe quelques programmes spécialisés tels que les logiciels de surtitrage Torticoli, Simultext ou Movitx qui permettent une plus grande précision et une souplesse accrue dans la production des surtitres et qui peuvent projeter simultanément des surtitres en plusieurs langues. Dans la plupart des théâtres 
canadiens, toutefois, le dispositif est fort simple: il comprend un ordinateur muni du logiciel PowerPoint, un projecteur et un écran. Le coût de production des surtitres est minime comparativement aux frais qu'engendre la traduction dramatique, laquelle nécessite le plus souvent la création d'un nouveau spectacle à partir d'un nouveau texte et avec une nouvelle équipe de production ${ }^{4}$. Subordonnés à la performance du texte, les surtitres contribuent à la théâtralité de l'œuvre dramatique et cette théâtralisation de la traduction impose des contraintes.

Tout comme à l'opéra, où «surtitles are subject to specific constraints of faithfulness and economy» (Mateo, 2004, p. 119), les surtitres pour le théâtre sont soumis à des contraintes de fidélité et d'économie qui sont différentes de celles de la traduction conventionnelle. Cette dernière éclipse le texte source et se substitue à lui de sorte qu'il demeure inconnu des lecteurs ou des spectateurs. Il est donc possible de s'éloigner du texte original pour rendre les dialogues acceptables ou plus appropriés pour le public visé. Cette appropriation peut être très accentuée dans le cas du texte de théâtre, puisque les dialogues doivent être immédiatement compréhensibles pendant le spectacle, sans l'aide de dictionnaires ou de notes explicatives. On ne peut prendre de telles libertés avec le surtitrage. Puisque l'auditoire entend le texte source et lit la traduction simultanément, cette dernière doit coller de près à l'original pour éviter de causer de la confusion ou de la distraction chez les spectateurs bilingues pouvant comprendre l'un et l'autre. À titre d'exemple, comparons les versions originale, traduite et surtitrée de la pièce d'Évelyne de la Chenelière, Bashir Lazhar ${ }^{5}$, créée au Théâtre d'aujourd'hui en janvier 2007. La pièce met en scène un enseignant d'origine algérienne récemment embauché dans une école québécoise. Dans l'extrait suivant, il corrige au tableau la dictée donnée à ses étudiants :

«Mes onze cent francs devaient suffire...» Devaient. Imparfait du verbe "devoir», à la troisième personne du pluriel: «a-i-e-n-t». Vous avez tendance à mettre un «s» dès qu'il s'agit du pluriel. Pourtant, en conjugaison, cette

4. Dans les contextes où les interprètes sont bilingues, la même production peut être présentée en alternance dans les langues source et cible. Cela est toutefois très rare. Ce fut le cas de Fort Mac de Marc Prescott, présenté en français et en anglais par le Théâtre Vice Versa Theatre à Winnipeg en 2011.

5. Voir Ladouceur et Liss (2011). 
règle ne s'applique pas. Un peu plus difficile: «Un ouvrage qui pût ...» non pas du verbe "puer» mais bien du verbe «pouvoir». (De la Chenelière, 2003, p. 56)

La traduction de la pièce signée par Morwyn Brebner est produite au Tarragon Theatre de Toronto en novembre 2008. Dans un souci de fluidité, la traductrice omet des énoncés difficiles à comprendre pour un public anglophone et séloigne considérablement du texte original lorsqu'il s'agit d'expliquer des règles de grammaire. Le même extrait se lit comme suit:

"My eleven hundred francs would have to last me three years." That's future conditional. Note the use of the auxiliary "would". A little more difficult : "A sphere of joy and silence in which-" No, not "witch" with a broom but "which" the non-restrictive pronoun. (De la Chenelière, 2008a, p. 5)

Invitée à Edmonton par L'UniThéâtre, la production québécoise originale y est présentée en octobre 2008 accompagnée de surtitres anglais. Afin de ne pas nuire à la communication, puisque le texte source oral et le texte cible écrit sont livrés simultanément et que le public francophone est en majorité bilingue, la version surtitrée doit viser la plus grande correspondance entre les deux messages. Voici le même extrait dans la version surtitrée:

"My eleven hundred francs were supposed to last me..."

That is the past tense. Make sure you use the past tense of the third person plural.

You have a tendency to put an "s" at the end of a verb to make it plural.

But for verbs that rule doesn't apply.

A little more difficult: "A work touted to draw public attention to me."

Not trout as in the fish, but tout as in to praise. (De la Chenelière, 2008b, diapo 143-145)

Soumis à des contraintes de fidélité et d'économie, les surtitres doivent par ailleurs se lire aisément et suivre de près le rythme de la représentation afin que la transmission des informations écrites et orales ait lieu simultanément. C'est pourquoi il est essentiel que la personne chargée de les projeter connaisse très bien la production, le rythme des dialogues et les surtitres correspondants afin de les synchroniser. 


\section{Surtitrage et création théâtrale}

La transmission live des surtitres pendant le spectacle permet d'élargir les fonctions que la traduction est appelée à remplir. Quand les surtitres n'offrent pas un substitut au message original, comme c'est le cas lorsque le spectateur connaît la langue source, ils offrent alors ce que Henrik Gottlieb appelle un «cognitive supplement, as is the case when audiences have simultaneous access to the translation and the original» (2012, p. 46). Ce supplément cognitif peut être mis à profit pour multiplier les messages transmis au public pendant le spectacle. Non plus imitation du texte source, la traduction prend alors une valeur créative en proposant de nouveaux messages qui s'ajoutent à ceux qui sont transmis par les dialogues sur scène. Ce fut le cas de Sex Lies et les Franco-Manitobains, pièce de Marc Prescott produite à Edmonton en 2009. Cette pièce bilingue met en scène une jeune femme et un cambrioleur qui sont francophones bilingues ainsi qu'un autre cambrioleur qui est anglophone unilingue, ce qui donne lieu à des quiproquos, des équivoques et des jeux de mots que seuls les spectacteurs bilingues peuvent comprendre. Les surtitres anglais, qui étaient en majorité fidèles aux dialogues en français, déviaient parfois de leur fonction première et offraient des messages indépendants.

Pour cette production, Marc Prescott, qui signait aussi la mise en scène, Shavaun Liss et moi, qui étions chargées des surtitres anglais ${ }^{6}$, avons adopté une approche qui accentuait la valeur ludique de la pièce et de sa mise en scène. La traductrice, assise sur la scène, activait son ordinateur au vu et au su du public et pouvait au besoin interagir avec les interprètes. Ainsi, pour pallier un trou de mémoire chez l'un deux, elle a pointé le doigt vers les surtitres où apparaissait l'information recherchée. Il lui est arrivé aussi de refuser de traduire des propos jugés vulgaires. Ainsi, lorsqu'un personnage s'est mis à hurler une série de jurons, le surtitre

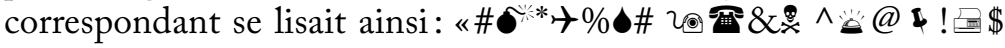
!» (Prescott, 2009, diapo 298). Les spectateurs ont alors été invités à interpréter cette traduction à leur façon, selon leurs propres références et préférences.

6. Il s'agit d'une expérimentation effectuée par Shavaun Liss sous ma supervision dans le cadre d'une recherche pour un mémoire de maîtrise intitulé Le surtitrage anglais du théâtre francophone de l'Ouest canadien: application et expérimentation (Liss, 2012). 
Par la suite, la traductrice s'est adressée au public pour commenter les propos d'un autre personnage. Pour cette production, Prescott avait révisé son texte initial et injecté un rap slang très prononcé dans les dialogues du cambrioleur anglophone. S'il fut bien apprécié des jeunes spectateurs, cet argot était toutefois difficilement compréhensible pour les non-initiés, ainsi que certains spectateurs nous l'ont fait remarquer après la première représentation. Pour remédier à la chose, il aurait fallu ajouter plusieurs surtitres anglais et s'assurer de leur synchronisation avant le prochain spectacle, ce que des contraintes de temps rendaient impossible. Consciente du problème mais incapable d'y remédier, la traductrice a quand même voulu accuser le coup en projetant ce surtitre à la représentation suivante pour accompagner la première réplique du cambrioleur anglophone: "If you don't understand what this guy is saying, don't worry-Neither does $50 \%$ of the rest of the audience. (This message brought to you by your friendly neighbourhood surtitle)»(Prescott, 2009, diapo 602). Le surtitre a alors transmis la voix hors champ de la traductrice, qui s'est superposée aux dialogues de la pièce jouée sur scène.

Enfin, les surtitres ont aussi mis à profit les ressources bilingues et biculturelles des Francophones en proposant parfois un message différent de celui qui était livré oralement, mais qui en offrait un équivalent culturel. Ainsi, lorsqu'une parodie d'un chant de Noël français bien connu a retenti sur scène, les surtitres ont donné à lire les paroles parodiées d'un autre chant anglais tout aussi connu. En voici un extrait, accompagné du surtitre correspondant:

Lui - C'est comme ça que ça se passe dans le temps des Fêtes/Tape le voleur, les filles pis le poêlon de galette/C'est comme ça que ça se passe avec un maudit fatiguant». (Prescott, 2001, p. 30).

Surtitre - Deck the robber for his folly / Fa-la-la-la-la la-la la la/With a frying pan in lieu of holly/Fa-la-la-la-la la-la la la. (Prescott, 2009, diapo 76)

Ici, seuls les spectateurs bilingues et biculturels ont pu apprécier la substitution et en rire.

L'emploi de surtitres ludiques ${ }^{7}$ a non seulement amplifié la dimension interculturelle de cette comédie qui explore les clichés, les défis et les écueils du bilinguisme, mais il a aussi investi la

7. Voir Nolette (2015) à ce sujet. 
traduction de fonctions accrues. En plus d'offrir des équivalences écrites au texte livré sur scène, les surtitres ont acquis une fonction créatrice en proposant de nouveaux messages qui ont multiplié les interprétations possibles du spectacle selon les ressources linguistiques des spectateurs. En fin de parcours, seuls les spectateurs bilingues ont pu comprendre la totalité des messages livrés sur scène et saisir l'ironie exprimée par la juxtaposition des informations divergentes livrées dans chaque langue. Ce procédé a eu pour conséquence de mettre en relief les avantages que procure le bilinguisme et de subvertir ainsi le discours monolingue dominant. Ces expérimentations ouvrent de nouvelles perspectives pour explorer le rapport qu'entretiennent les langues et les cultures mises en contact par la traduction dans les arts de la scène ${ }^{8}$. Ce rapport, déjà à l'œuvre dans la conception des surtitres, agit aussi sur leur réception auprès des publics visés.

\section{Surtitrage et cohabitation des langues}

La traduction théâtrale par surtitres se distingue aussi de la traduction dramatique par le haut degré de visibilité qui la caractérise (Ladouceur, 2013b). Alors que l'opération traduisante peut demeurer invisible dans le cas de la traduction conventionnelle, elle s'affiche nécessairement dans la traduction théâtrale puisque les textes source et cible sont transmis "parallel to each other, that is, at the same time and place, and overtly» (Griesel, 2005, p.6). Tout comme le sous-titrage, le surtitrage «can be described as a foreignizing, or overt, type of translation» (Gottlieb, 2004, p. 90), «retaining the original version, thus laying itself bare to criticism from everybody with the slightest knowledge of the source language» (ibid., p. 102). Cette visibilité des surtitres est le reproche le plus fréquent qu'on leur adresse:

Supertitles, forcing spectators to shift their focus, even if momentarily, away from the stage, are much more actively disruptive, since they are directly competing with other stimuli to the visual channel, leaving unimpeded the auditory channel. (Carlson, 2006, p. 197)

8. L'usage de surtitres créatifs dans En français comme en anglais, It's Easy to Criticize de Jacob Wren, présenté à l'École nationale de théâtre du Canada en octobre 2011, avait pour effet de multiplier les messages, dont certains nétaient accessibles qu'aux spectateurs bilingues (voir Ladouceur, 2013a). 
Afin de limiter cette interférence, les surtitres doivent éliminer l'information non essentielle et livrer le message de la façon la plus succincte possible ${ }^{9}$, ce qui pourra toutefois être perçu comme un manque de fidélité par certains spectateurs bilingues.

Parce qu'il affiche le travail de la traduction, qui est transmise simultanément au texte source, le surtitrage impose une cohabitation des langues qui peut influer sur la réception des surtitres auprès des spectateurs non visés par la traduction. Non plus restreinte uniquement aux destinataires du texte traduit, comme c'est le cas pour la traduction conventionnelle, la traduction par surtitrage est transmise sans discrimination aux destinataires de la traduction et aux destinataires de l'œuvre originale pendant le spectacle. Si elle invite à une rencontre collégiale entre les locuteurs de la langue source et de la langue cible, la cohabitation imposée par le surtitrage peut aussi exacerber des tensions déjà existantes entre les groupes linguistiques concernés. Comme le souligne Sirkku Aaltonen, "[a] theatre production is always closely tied to its audience in a particular place at a particular point in time » (2000, p. 58). Lieu de rassemblement par excellence pour une communauté, le théâtre est investi d'une fonction identitaire qu'il ne partage pas avec l'opéra. Ancré dans son contexte socioculturel immédiat, le spectacle théâtral est un outil privilégié d'exploration et d'affirmation d'une identité culturelle, à plus forte raison quand cette identité est menacée.

Au Canada, l'usage des surtitres est répandu surtout dans les théâtres francophones situés à l'ouest du Québec et dans le cadre de festivals internationaux qui présentent des spectacles en diverses langues, comme le Festival TransAmériques à Montréal et le Gateway Pacific Theatre Festival ${ }^{10}$ à Vancouver. Les théâtres franco-québécois y font rarement appel. Récemment, le théâtre de La Manufacture a innové en présentant pendant la saison régulière des spectacles en traduction française accompagnés de surtitres anglais. Cette pratique a été inaugurée en novembre 2012 avec En ce moment, une traduction québécoise signée par Maryse Warda du texte de l'auteure irlandaise Deirdre Kinahan intitulé Moment.

9. Par exemple, il est préférable d'utiliser des chiffres et non des lettres pour indiquer les nombres.

10. Dans le cadre de ce festival, certains spectacles sont présentés en cantonnais avec surtitres chinois et anglais. Voir le site du festival: https:// www.gatewaytheatre.com/. 
Jusqu'à présent, la troupe a appliqué cette politique exclusivement aux pièces d'auteurs anglophones traduites et présentées en français. Il s'agit toutefois de rares incursions des surtitres anglais sur les scènes franco-québécoises. Par contre, les troupes angloquébécoises y ont recours de plus en plus fréquemment afin de rejoindre le vaste auditoire francophone qui les entoure. Depuis 2009, la troupe montréalaise bilingue Théâtre Porte-Parole Theatre intègre des surtitres français à ses spectacles (Ladouceur, 2014). Le Centaur Theatre ainsi que le Infinitheatre, tous deux situés à Montréal, offrent aussi depuis peu des spectacles avec surtitres français. Ailleurs au Canada, toutefois, les théâtres anglophones n'incluent pas de surtitres français dans leurs productions. Jouissant d'un nombre élevé de spectateurs potentiels, ils ne ressentent pas le besoin de rejoindre un public francophone peu nombreux. Il s'agit donc d'un mode de traduction pratiqué presque exclusivement par des compagnies œuvrant dans une langue minoritaire et qui désirent rejoindre le public plus large des locuteurs de la langue dominante.

De 2008 à 2013, mes recherches sur le surtitrage mont amenée à produire les surtitres anglais de treize spectacles présentés à L'UniThéâtre, la seule compagnie professionnelle francophone en Alberta $^{11}$. Cette collaboration entre l'université et la communauté francophone d'Edmonton a permis à des étudiants-chercheurs d'acquérir une formation pratique du surtitrage et de participer à des recherches sur le sujet tout en contribuant au rayonnement du théâtre franco-albertain. Ces productions mont fourni l'occasion non seulement de concevoir et de présenter des surtitres, mais aussi d'étudier leur réception auprès des différents destinataires $\mathrm{du}$ spectacle, locuteurs des langues source et cible, unilingues ou bilingues.

\section{Destinataires et réception des surtitres}

L'emploi des surtitres dans les théâtres franco-canadiens modifie la composition de l'auditoire. Outre les Francophones, à qui le spectacle original est destiné, l'auditoire inclut aussi des Anglophones, à qui les surtitres sont destinés. Pour ces derniers, qui sont en majorité unilingues dans l'Ouest canadien, les surtitres sont un outil indispensable pour comprendre des dialogues en

11. Le Théâtre à Pic, fondé à Calgary en 2011, se qualifie de «coopérative théâtrale francophone». 
français. Ces spectateurs anglophones incluent non seulement des amateurs de théâtre, mais aussi des créateurs, interprètes, artisans et agents culturels anglophones qui peuvent ainsi avoir accès aux productions en français. Pour les artistes œuvrant dans l'extrême marge de la francophonie canadienne, la rencontre avec leurs pairs anglophones suscite des échanges et des collaborations qui brisent l'isolement auquel l'éloignement les contraint et contribue à nourrir un discours critique nécessaire pour l'avancement d'une pratique artistique. En soumettant ainsi leurs productions à l'appréciation de leurs pairs anglophones, les artistes franco-canadiens s'inscrivent dans une dynamique essentielle à la croissance artistique et communautaire. Comme le souligne Lucie Hotte, «produire des œuvres artistiques en contexte minoritaire qui soient dignes de concurrencer les œuvres produites ailleurs témoigne de la vitalité d'une communauté, encourage les jeunes à devenir à leur tour des créateurs» (2013, p. 16). Ainsi, lorsque L'UniThéâtre a reçu en 2013 le «Elizabeth Sterling Haynes Award for its outstanding contribution to Edmonton theatre» (site web, The Elizabeth Sterling Haynes Awards ${ }^{12}$ ), le communiqué de presse précisait que "l'initiative de L'UniThéâre d'offrir des surtitres en anglais de ses représentations depuis quelques années a créé un grand impact dans le milieu théâtral d'Edmonton» (L'UniThéâtre, 2013). En augmentant l'accessibilité à leurs productions, le surtitrage permet aux troupes œuvrant dans une langue minoritaire d'acquérir une reconnaissance et une légitimité qui autrement leur ferait défaut.

Pour les Francophones qui assistent aux spectacles surtitrés en anglais et à qui les surtitres ne sont pas destinés, les usages peuvent varier. Ceux qui sont moins exposés au français dans la vie quotidienne ou moins à l'aise avec les accents des interprètes font appel aux surtitres anglais de façon sporadique pour comprendre certains dialogues livrés en français sur scène (voir Liss, 2012 et Pridmore-Franz, 2017). Pour ceux qui sont plus à l'aise en français et peuvent facilement comprendre les dialogues, les surtitres deviennent parfois source de distraction, car la tentation est grande de comparer les messages transmis dans chaque langue pendant le spectacle. Cet intérêt que plusieurs spectateurs bilingues portent aux surtitres peut causer beaucoup d'interférence. Dans son étude

12. Depuis 1987, les prix Elizabeth Sterling Haynes sont décernés chaque année à Edmonton pour l'excellence dans le domaine du théâtre. 
des mouvements oculaires des spectateurs entre l'image et les soustitres, Elisa Ghia a comparé les temps de lecture des sous-titres qui offrent une traduction littérale et ceux qui ne reproduisent pas littéralement le texte (voir Pridmore-Franz, 2017). Elle a remarqué que, pour les spectateurs qui comprennent les messages source et cible, le temps de lecture est plus long lorsque les traductions ne sont pas littérales, car les spectateurs les comparent alors plus attentivement avec le message source:

non-literal translation of the dialogue may intensify the mechanisms of mapping and comparison between ST [source text] and TT [target text], as well as stimulate a deeper processing of the input components. (Ghia, 2012, p. 176)

Fotios Karamitroglou explique:

[t]his occurs because of the constant presence of an inherently operating checking mechanism in the brain of the viewers which raises the suspicions that the translation of the original text is not "properly" or "correctly" rendered in the subtitles, every time word-for-word translations for such items are not spotted. (1998, n.p.)

Il y aurait donc de la part des locuteurs bilingues une méfiance envers la traduction lorsquelle ne reproduit pas le texte source mot à mot, ce qui augmente le temps de lecture des surtitres et accentue l'interférence qu'ils peuvent causer.

Si la majorité des spectateurs bilingues acceptent ou tolèrent les surtitres anglais parce qu'ils servent une bonne cause, soit le rayonnement de la culture francophone, il y a toutefois certains irréductibles qui s'y opposent farouchement, les critiquent publiquement et refusent d'assister aux spectacles surtitrés. Ces réfractaires au surtitrage appartiennent surtout à la génération des pionniers du théâtre francophone en Alberta. Ils ont travaillé d'arrache-pied à mettre en place des troupes francophones, telles le Théâtre Français d'Edmonton en 1967, et à produire des pièces en français à une époque où les moyens de production et les appuis financiers accordés à la culture franco-canadienne étaient pratiquement inexistants (voir Godbout, Ladouceur et Allaire, 2012). Pour eux, le théâtre est un outil privilégié de résistance à la domination anglaise, et la présence des surtitres anglais ouvre une 
brèche dans la forteresse qu'ils ont érigée à l'intérieur des murs du théâtre pour contrer l'anglicisation et affirmer publiquement la présence d'une langue française autonome et souveraine. Encore aujourd'hui, dans les petites communautés francophones éloignées des grands centres culturels et de leurs réseaux institutionnels, le théâtre demeure l'art communautaire par excellence, un art vivant pratiqué intensément par des gens désireux d'exercer leur langue dans une activité culturelle qui la met en valeur.

Cette résistance aux surtitres anglais dans les théâtres francocanadiens signifie que leur présence est perçue comme dangereuse en ce qu'elle fragilise encore davantage une langue minoritaire déjà fragile. La cohabitation des langues par l'entremise des surtitres oblige le français à partager la scène avec l'anglais dans un lieu consacré pourtant à la promotion et à la protection du français. Les deux langues se trouvent ainsi à rivaliser entre elles et, pour plusieurs spectateurs, l'anglais des surtitres qui dominent la scène se substitue alors au français comme langue de communication, soulignant la précarité d'une langue française qui ne peut plus assumer seule la communication et doit être relayée par l'anglais. $\mathrm{Si}$ le surtitrage anglais peut procurer des avantages financiers et symboliques aux compagnies théâtrales franco-canadiennes, en contribuant au rayonnement et à la reconnaissance de leur travail, il peut aussi causer préjudice au français et remettre en cause la vocation culturelle de ces théâtres fonctionnant dans une langue française minorisée.

\section{Conclusion}

Le surtitrage du spectacle théâtral se distingue dans le champ de la traduction audiovisuelle parce que la traduction est appliquée ici à un art vivant qui occupe une fonction particulière au sein d'une communauté culturelle donnée. Et cette fonction est encore plus accentuée quand l'identité culturelle de la communauté visée est menacée. S'affichant à tous les spectateurs sans distinction, rassemblés dans l'enceinte du théâtre, la traduction peut être ressentie comme une transgression portant atteinte à l'intégrité de l'expérience culturelle vécue par les destinataires de l'œuvre originale. En contexte minoritaire, l'intrusion de la langue dominante dans l'enceinte du théâtre a pour effet de raviver l'insécurité linguistique des locuteurs de la langue minoritaire. Cette condition minoritaire est d'ailleurs mise en relief par l'absence de surtitres sur les scènes 
anglo-canadiennes alors qu'ils sont devenus courants dans les théâtres franco-canadiens, une pratique qui illustre bien l'asymétrie des langues officielles du Canada et l'inégalité du rapport de force qu'elles entretiennent.

\section{Références}

Aaltonen, Sirkku (2000). Time-Sharing on the Stage: Drama Translation in Theatre and Society. Topics in Translation 17. Clevedon, Multilingual Matters.

Association des théâtres francophones du Canada. [http://atfc.ca/index. cfm?Id=38960\&Sequence_No=38960\&niveau=3\&Voir=bulletin_ article].

Bartoll, Eduard (2011). «The Surtitling in Catalan of Classic Foreign Theatre Plays». In L. Incalcaterra McLoughin, M. Biscio, M. A. Ní Mhainnín, dir. Audiovisual Translation. Subtitles and Subtitling: Theory and Practice. Bruxelles, Peter Lang, p. 87-109.

Bassnett, Susan (1991). «Translating for the Theatre: The Case Against Performability». TTR, 4, 1, p. 99-111.

Burton, Jonathan (2010). «The Joy of Opera: The Art and Craft of Opera Subtitling and Surtitling». In L. Bogucki et K. Kredens, dir. Perspectives on Audiovisual Translation, Francfort, Peter Lang, p. 180188.

Carlson, Marvin (2006). Speaking in Tongues: Language at Play in the Theatre. Ann Arbor, The University of Michigan Press.

De la Chenelière, Évelyne (2003). Au bout du fil; Bashir Lazhar. Paris, Éditions Théâtrales.

De la Chenelière, Évelyne (2008a). Bashir Lazhar, trad. anglaise Morwyn Brebner, version manuscrite fournie par la traductrice.

De la Chenelière, Évelyne (2008b). Bashir Lazhar, surtitres anglais de Shavaun Liss sous la supervision de Louise Ladouceur, d'après la traduction de Morwyn Brebner.

Dewolf, Linda (2003). «La place du surtitrage comme mode de traduction et vecteur d'échange culturel pour les arts de la scène». Recherches théâtrales au Canada, 24, 1-2, p. 92-108.

Gambier, Yves, (2004). «La traduction audio-visuelle: un genre en expansion». Meta, 49, 1, p. 1-11.

Ghia, Elisa, (2012). «The Impact of Translation Strategies on Subtitle Reading». In E. Perego, dir. Eye-tracking in Audiovisual Translation. Scienze dell'Antichità, Filologico-Letterarie e Storico-Artistiche 895. Rome, Aracne Éditrice, p. 157-182.

Godbout, Laurent, Louise Ladouceur et Gratien Allaire (2012). Plus d'un siècle sur scène: histoire du théâtre francophone en Alberta de 1887 
à 2008. Edmonton, Institut du patrimoine des francophones de l'Ouest canadien.

Gottlieb,Henrik (2004). «Language-political Implications of Subtitling». In P. Orero, dir. Topics in Audiovisual Translation, Amsterdam/ Philadelphie, John Benjamins, p. 83-100.

Gottlieb, Henrik (2012). «Subtitles: Readable dialogue?» In E. Perego, dir. Eye-tracking in Audiovisual Translation. Scienze dell'Antichità, Filologico-Letterarie e Storico-Artistiche 895. Rome, Aracne Éditrice, p. 37-81.

Griesel, Yvonne (2005). «Surtitles and Translation. Towards an Integrative View of Theatre Translation». EU High Level Scientific Conference Series. MuTra 2005 - Challenges of Multidimensional Translation: Conference Proceedings. [http://www.euroconferences. info/proceedings/2005_Proceedings/2005_Griesel_Yvonne.pdf].

Hotte, Lucie (2013). «Artiste, animateur culturel ou médiateur culturel? Le rôle des artistes dans les communautés francophones du Canada». Minorités linguistiques et société/Linguistic Minorities and Society, 3, p. 7-18.

Karamitroglou, Fotios. (1998). «A Proposed Set of Subtitling Standards in Europe». Translation Journal, 2, 2, n.p. [http://translationjournal. net/journal/04stndrd.htm].

Ladouceur, Louise (2005). Making the Scene: la traduction du théatre d'une langue officielle à l'autre au Canada. Québec, Nota bene.

Ladouceur, Louise (2013a). «Exploring a Bilingual Aesthetics through Translation in Performance». In P. Ambrosi, S. Bigliazzi et P. Kofler, dir. Theatre Translation in Performance. New York et Londres, Routledge, p. 111-129.

Ladouceur, Louise (2013b). «Surtitles Take the Stage in FrancoCanadian Theatre». Target, 25, 3, p. 343-364.

Ladouceur, Louise (2014). «Bilingual Performance and Surtitles: Translating Linguistic and Cultural Duality in Canada». Linguistica Antverpiensia New Series - Themes in Translation Studies, 13, p. 4560. [https://lans-tts.uantwerpen.be/index.php/LANS-TTS/article/ view/54].

Ladouceur, Louise et Shavaun Liss (2011). «Identité bilingue et surtitres ludiques dans les théâtres francophones de l'Ouest canadien». Francophonies d'Amérique, 32, p. 171-186.

Laliberté, Michèle (2017). «Un surtitrage fonctionnel, artistique ou intermédial? Réalités théâtrales multilingues à l'ère numérique». Recherches théâtrales au Canada, 38, 1, p. 75-92.

Liss, Shavaun (2012). Le surtitrage anglais du théatre francophone de l'Ouest canadien: application et expérimentation. Mémoire de maitrise, 
Campus Saint-Jean, Université de l'Alberta.

L'UniThéâtre (2013). Célébré par ses pairs anglophones, communiqué de presse, 25 juin.

Mateo,Marta (2004).«Reception, Text and Context in the Study of Opera Surtitles». In Y. Gambier, M. Shlesinger et R. Stolze, dir. Doubts and Directions in Translation Studies. Amsterdam/Philadelphie, John Benjamins, p. 169-182.

Nolette, Nicole (2015). Jouer la traduction. Théâtre et hétérolinguisme au Canada francophone. Ottawa, Presses de l'Université d'Ottawa.

Pavis, Patrice (1989). "Problems of Translation for the Stage: Interculturalism an Post-modern Theatre». In $\mathrm{H}$. Scolnicov et P. Holland, dir. The Play Out of Context: Transferring Plays from Culture to Culture. Cambridge, Cambridge University Press.

Prescott, Marc (2001). Big; Bullshit; Sex, Lies et les Franco-Manitobains. Saint-Boniface, Les Éditions du Blé.

Prescott, Marc (2009). Sex, Lies et les Franco-Manitobains, surtitres anglais de Shavaun Liss et Louise Ladouceur, Théâtre au Pluriel, Université de l'Alberta, 5-6 novembre.

Pridmore-Franz, Milane (2017). A Study on the Audience Reception of Theatre Surtitles: Surtitling in a Francophone Minority Context in Canada and the Language Learning Potentials of Theatre Surtitles. Mémoire de maitrise, Dep. of Modern Languages and Cultural Studies, University of Alberta.

The Elizabeth Sterling Haynes Awards. [http://www.sterlingawards. com/Sterling_Awards/Home.html].

\author{
Louise Ladouceur \\ Université de l'Alberta \\ 8406 - 91 Street \\ Edmonton (Alberta) T6C 4G9 \\ CANADA \\ louise.ladouceur@ualberta.ca
}

